\title{
Link between Hyperhomocysteinemia, Glycemic and Lipid Profiles, in Type 2 Diabetes in Côte d'Ivoire
}

Research Article

\author{
Diagou JJ ${ }^{1,2 *}$, Ocho-Anin Atchibri AL ${ }^{1}$, Gbogouri GA ${ }^{1}$, Acka F $F^{4}$, Ake $\mathrm{M}^{3}$, N'guessan-Blao R², Zanon $\mathrm{TG}^{2}$, Kouassi $\mathrm{D}^{2,3,4}$ \\ ${ }^{1}$ Laboratory of Nutrition and Food Safety, UFR of Sciences and Food Technologies, Nangui Abrogoua University, Côte d'Ivoire. \\ ${ }^{2}$ Laboratory of Research and Medical Biology, National Institute of Public Health (INSP), Abidjan, Côte d'Ivoire. \\ ${ }^{3}$ Nutrition Research Center, INSP, Côte d'Ivoire. \\ ${ }^{4}$ Diabetic Center of Abidjan (CADA), INSP, Côte d'Ivoire.
}

Abstract

The hyperhomocysteinemia is associated with the development and the accentuation of the cardiovascular complications of the type 2 diabetes. Several studies had established a link between the hyperhomocysteinemia, the dyslipidemias and the atherosclerosis. In Côte d'Ivoire, no study has explored this link. The aim of this study was to evaluate the correlative link between the hyperhomocysteinemia, the glycemia and the lipid profiles. This study was realized in 200 volunteer type 2 diabetics, regularly monitored at the Diabetic Center of Abidjan. The homocysteine, the glycemia and the lipid parameters were assayed by enzymatic methods on the biochemistry automaton Cobas c-311. The present study has revealed that the hyperhomocysteinemia was strongly correlated with the duration of the type 2 diabetes $(\varrho=+0.999, \mathrm{p}<0.05)$. The study did not show a correlation between the hyperhomocysteinemia and the glycemia $(\varrho=-0.097, \mathrm{p}<0.05)$; whether during the balanced glycemia $(\varrho=-0.057$, $\mathrm{p}>0.05)$ or during the very unbalanced glycemia $(\geq 1.26 \mathrm{~g} / \mathrm{L})(\varrho=+0.0237 ; \mathrm{p}>0.05)$. This could partly be explained by the effect of the clinical and nutritional treatment in our diabetics. However, when the glycemia is regulated at the state of hyperglycemia, we had observed a strong correlation between the hyperhomocysteinemia and the hyperglycemia $(\varrho=+0.975, \mathrm{p}$ $<0.05)$. The study did not show a correlation between the hyperhomocysteinemia and the lipids in our diabetics under anti-lipid treatment: triglycerides $(\varrho=-0.132, \mathrm{p}<0.05)$; total cholesterol $(\varrho=-0.124, \mathrm{p}<0.05)$; LDL cholesterol $(\varrho=-0.105, \mathrm{p}<0.05)$; HDL cholesterol $(\varrho=+0.107, \mathrm{p}<0.05)$. The quality of the glycemic and lipid balance and the duration of diabetes, can affect the effect of hyperhomocysteinemia in the development of the cardiovascular diseases.

Keywords: Hyperhomocysteinemia; Glycemia; Lipid Profile; Correlative Link; Type 2 Diabetes.

\section{Introduction}

The homocysteine is a non-proteinogenic sulfur-containing amino acid, intermediate in the metabolism of methionine [1]. It is synthesized by all the cells of the body and is catabolized along two metabolic pathways: the transsulfuration pathway and the pathway of remethylation [4]. This amino acid is an important indicator in the risk assessment of the cardiovascular diseases [2]. Under normal physiological conditions, the homocysteine is metabolized as soon as it is formed, so that its plasma concentration remains very low [11]. The plasma concentration of homocysteine in a normal subject is generally between 5 and 15 $\mu \mathrm{mol} / 1$ [4]. However, when its metabolization is done less well and less rapidly, the homocysteine tends to accumulate in the blood: it is the hyperhomocysteinemia [3]. The hyperhomocysteinemia is an abnormal accumulation of plasma homocysteine [3]. The nutritional deficiencies acquired in vitamins $B$ and the genetic mutations mainly affecting the methionine synthase, the methylenetetrahydrofolate reductase and the cystathionine $\beta$-synthase, are the main pathways leading to the hyperhomocysteinemia $[3,15$, 23]. Addiction to an excessive diet of methionine-rich protein leads to the hyperhomocysteinemia [24-26]. Low consumption of fruits and vegetables (rich in B vitamins and antioxidants) and low consumption of multivitamins are implicated in the occurrence of the hyperhomocysteinemia [25]. The factors such as smoking, alcoholism, coffee consumption, also lead to the hyperhomocysteinemia [27] by inference with the metabolism of homocysteine. Several physiological factors are related to the

\footnotetext{
*Corresponding Author:

Diagou Jean Jacques,

Laboratory of Nutrition and Food Safety; UFR of Sciences and Food Technologies; Nangui Abrogoua University (02, BP 801 Abidjan 02). Laboratory of Research and Medical Biology;

National Institute of Public Health (INSP), Abidjan (BP V 47 Abidjan). Diabetic Center of Abidjan (CADA), INSP, Côte d'Ivoire.
}

E-mail: vitalis.saturne@yahoo.fr

Received: March 08, 2018

Accepted: April 17, 2018

Published: April 20, 2018

Citation: Diagou JJ, Ocho-Anin Atchibri AL, Gbogouri GA, Acka F, Ake M, N'guessan-Blao R, et al., Link between Hyperhomocysteinemia, Glycemic and Lipid Profiles, in Type 2 Diabetes in Côte d'Ivoire. Int J Diabetol Vasc Dis Res.. 2018;6(2):217-222. doi: http://dx.doi.org/10.19070/2328-353X-1800044

Copyright: Diagou JJ ${ }^{\circ} 2018$. This is an open-access article distributed under the terms of the Creative Commons Attribution License, which permits unrestricted use, distribution and reproduction in any medium, provided the original author and source are credited. 
hyperhomocysteinemia: age, gender, and metabolic alterations associated with aging $[27,28]$. The drugs: anti-folic, antiepileptic, anti-estrogenic, lipid-lowering drugs, some antibiotics, and some oral contraceptives, etc., increase the plasma homocysteine levels $[23,28]$.

The hyperhomocysteinemia is an independent cardiovascular risk factor [5]. Its presents with the diabetes a deleterious synergy [29]. The hyperhomocysteinemia is associated with the development and the accentuation of the cardiovascular complications of the type 2 diabetes [5]. Its deleterious effect is particularly potent in the diabetics than in non-diabetics, leading to the emergence of the micro and macro-vascular atherotrombotic diseases [6] and to a $90 \%$ mortality rate over 5 years [4].

Several studies have established a link between the hyperhomocysteinemia, the dyslipidemias and the atherosclerosis [7]. However, those linking the hyperhomocysteinemia to the lipid profiles are limited and often contradictories [7]. In Côte d'Ivoire, no study was devoted to the correlation between the hyperhomocysteinemia, the glycemia and the lipid parameters in the type 2 diabetic subjects. The present work consisted to investigating the link between the hyperhomocysteinemia, the glycemia and the lipid profiles in a type 2 diabetic population under anti-diabetic, anti-lipid and dietary therapy. Specifically, it was to study the correlation between the hyperhomocysteinemia, the duration of the diabetes, the glycemia and the lipid parameters. This, in order to appreciate the effect of independence or synergy of the hyperhomocysteinemia vis-a-vis the metabolism of the carbohydrates and the lipids, during the development of the cardiovascular diseases of the type 2 diabetes.

\section{Methodology}

\section{Type of study}

This was a descriptive and cross-sectional study.

\section{Study Population}

This study was conducted from September to December 2016, and has involved 231 subjects. The type 2 diabetic population was composed of 200 subjects, including 122 women and 78 men, aged from 23 to 86 years. They were regularly monitored at the Diabetic Center of Abidjan. The diabetic subjects included different Ivorian and foreign ethnic groups, composed of 171 nationals and 29 non-nationals. The non-diabetic control population consisted of 31 healthy volunteers, including 18 men and 13 women, aged from 22 to 50 years. They were recruited from the health staff of the National Institute of Public Health (INSP) of Abidjan - Côte d'Ivoire and among other people outside the institute.

The study protocol has been approved by the Pasteur Institute of Côte d'Ivoire. All the subjects were informed of the purpose of the study and have given their informed consent of participation.

\section{Blood Sampling and Determination of Biochemical Parameters}

The venous blood sampling of the fasting subjects (12 hours) was performed, in a semi-sitting position, in the sampling room of the INSP Medical Research and Biology Laboratory. The blood was collected in $5 \mathrm{ml}$ Vacutainer tubes, immediately placed in a cooler between the collection and the centrifugation. The EDTA (Ethylene diamine tetra-acetic acid) tubes (purple), for the determination of the homocysteine; the $\mathrm{NaF}$ (sodium fluoride) tubes (gray), for the determination of the glycemia; and the dry tubes (red) for the lipid assay. After the centrifugation of the tubes at $3000 \mathrm{rpm}$ for 10 minutes, the plasmas were aliquoted and stored at $-20^{\circ} \mathrm{C}$. At the time of the assays, the plasma samples were thawed at the room temperature and homogenized. The homocysteinemia, the glycemia and the lipid parameters were assayed by enzymatic methods on the biochemistry automaton Cobas c-311 (Roche Diagnos-tics). The homocysteine, by the enzymatic cycling method, using the Cobas-Integras kit, reference No: 0538415190 (Germany). The glycemia, by the glucose oxidase method (GOP-PAP), using the Cobas-Integras kit, reference No: 04404483190 (Germany). The triglycerides, by the glycerolphosphate oxidase method (GPO-PAP), using the Cobas-Integras kit, reference No: 20767107322 (Germany). The total cholesterol, by the cholesterol oxidase method (CHOD-PAP), using the Cobas-Integras kit, reference No: 03039773190 (Germany). The HDLs were evaluated by the enzymatic colorimetric method (Direct Homogeneous Test for HDL-Cholesterol), using the Cobas-Integras kit, reference No: 04399803190 (Germany). The LDLs were determined by the formula of Fredwald et al., [30]. The biochemical reference values of the glycemia and the lipids are those of the Ivorian adult presumed healthy, in force at INSP-Abidjan and coming from the study of Yapo et al., [8]. The reference value of homocysteinemia is that of Faeh et al., [9], adopted in 2001 by the Homocysteine Group of France.

\section{Statistical Analyses}

The data were recorded and then processed by the Excel 2013 application. The biochemical variables (homocysteinemia, glycemia, lipids) were expressed under the form of mean \pm standard deviation. These quantitative variables were evaluated by the Fisher test for the equality of variances and the Student's test for the comparison of means. The correlation coefficient $\varrho$ of Spearman was used to establish the correlative link between the hyperhomocysteinemia, the glycemia and the lipid parameters. The presumption of the correlative link was evaluated by the correlation coefficient improvement test (A), according to the formula: $A(\%)=[1-\sqrt{ }(1-\varrho 2)]^{* 100)}[10]$. For these tests, the significance was allowed for $\mathrm{p}<0.05$.

\section{Results}

\section{The Biological Variables of the Hyperhomocysteinemic Type 2 Diabetics and the Controls}

The biological variables of the hyperhomocysteinemic diabetics and the controls are presented in table 1. A significant difference existed between the hyperhomocysteinemic and the control group: for the homocysteinemia, the glycemia and the lipid parameters. However, in these diabetics, the total cholesterol, the triglycerides, the LDLs cholesterol and the HDLs-cholesterol values were within the normal ranges of reference. 
Correlation Studies between the Hyperhomocysteinemia, the Duration of Diabetes and the Glycemia

The relational study between the hyperhomocysteinemia and the duration of the type 2 diabetes is presented in table 2 . It has revealed the existence of a significant, positive and very strong correlation (Spearman correlation coefficient $(\varrho)=+0.999$; presumption of correlative link: $99.80 \%$ ). The hyperhomocysteinemia was strongly correlated with the duration of diabetes.

The correlation study between the hyperhomocysteinemia and the glycemia is presented at the table 3 . The study has revealed a significantly negative and low correlative correlation $(\varrho=-0.097$; correlation link presumption: $0.5 \%$ ). When the glycemia is balanced $(\leq 1.10 \mathrm{~g} / \mathrm{L})$, the correlation is negative, low and not significant.
When the glycemia is regulated at the state of hyperglycemia $(1.10<$ glycemia $<1.26 \mathrm{~g} / \mathrm{L})$, the correlation bet-ween the hyperhomocysteinemia and the hyperglycemia was significant and positive $(\varrho=+0.975$; correlative link presumption: $77.70 \%)$. In addition, when the glycemia is purely diabetic (glycemia $\geq$ $1.26 \mathrm{~g} / \mathrm{L})$, the correlation between the hyperhomocysteinemia and the glycemia was not significant.

\section{Correlation Studies between the Hyperhomocysteinemia and the Lipid Parameters}

The relationship between the hyperhomocysteinemia and the lipids is presented at the table 4 . The Spearman correlation coefficients, and the correlative presumption between hyperhomocysteinemia and the lipids were very weak (table4).

Table 1. Mean biological values of diabetics and controls.

\begin{tabular}{|c|c|c|c|c|}
\hline & $\begin{array}{c}\text { Hyperhomocysteinemic } \\
\text { diabetics } \mathbf{n = 7 3}\end{array}$ & $\begin{array}{c}\text { Non Diabetic controls } \\
\mathbf{n}=\mathbf{3 1}\end{array}$ & $\begin{array}{c}\text { Reference } \\
\text { values }\end{array}$ & $\mathbf{P}$ \\
\hline Glyc & $1.53 \pm 0.81[0.78-4.63]$ & $0.85 \pm 0.09[0.71-1.04]$ & $0.7-1.10 \mathrm{~g} / \mathrm{L}$ & $<0.05$ \\
\hline CT & $2.07 \pm 0.60[0.86-4.59]$ & $1.94 \pm 0.35[1.39-2.62]$ & $1.06-2.50 \mathrm{~g} / \mathrm{L}$ & $<0.05$ \\
\hline HDL & $0.43 \pm 0.11[0.12-1.35]$ & $0.66 \pm 0.16[0.32-0.91]$ & $0.26-0.70 \mathrm{~g} / \mathrm{L}$ & $<0.05$ \\
\hline LDL & $1.42 \pm 0.57[0.37-2.55]$ & $1.11 \pm 0.34[0.47-1.69]$ & $0.55-1.30 \mathrm{~g} / \mathrm{L}$ & $<0.05$ \\
\hline TG & $1.04 \pm 0.33[0.39-1.77]$ & $0.77 \pm 0.27[0.36-1.47]$ & $0.30-1.20 \mathrm{~g} / \mathrm{L}$ & $<0.05$ \\
\hline HCY & $14.84 \pm 3.26[12.00-28.78]$ & $10.74 \pm 2.38[6.07-15.67]$ & $<12 \mu \mathrm{mol} / \mathrm{L}$ & $<0.05$ \\
\hline
\end{tabular}

P: significance; [Min-Max]: median value; Min: minimum; Max: maximum; HCY: homocysteinemia; Glyc.: Glycemia; CT: total cholesterol; TG: triglycerides; LDL: low density lipoprotein cholesterol; HDL: high density lipoprotein cholesterol.

Table 2. Correlation between the hyperhomocysteinemia and the duration of the type 2 diabetes.

\begin{tabular}{|c|c|c|c|c|c|}
\hline $\begin{array}{c}\text { (n= 73) } \\
\text { HHcy }\end{array}$ & \multicolumn{2}{|c|}{$\varrho \mathbf{9 5 \% \text { CI }}$} & $\begin{array}{c}\text { A (\%) } \\
\text { (Presumption } \\
\text { of link correlation) }\end{array}$ & $\begin{array}{c}\text { *Condition of existence } \\
\text { of the correlative link : } \\
\text { A }>\mathbf{5 0 \%} \varrho>\mathbf{0 . 8 7}\end{array}$ & $\mathbf{P}$ \\
\hline Duration of diabetes & +0.999 & $0.96-1$ & 99.80 & $\begin{array}{c}\mathrm{A}>50 \% \\
\varrho>0.87\end{array}$ & $<0.05$ \\
\hline
\end{tabular}

P: significance; HHcy: hyperhomocysteinemia; @: Spearman correlation coefficient; CI: confidence interval; A: correlation coefficient improvement expressing the presumption of the correlative link $\left(A=[1-\sqrt{ }((1-\varrho 2))]^{* 100)}[10]\right.$.

Table 3. Correlation between the hyperhomocysteinemia and the glycemia.

\begin{tabular}{|c|c|c|c|c|c|}
\hline $\begin{array}{l}(n=73) \\
\text { HHcy }\end{array}$ & \multicolumn{2}{|c|}{ @ 95\% CI } & $\begin{array}{c}\text { A (\%) } \\
\text { (Presumption } \\
\text { of link correlation) }\end{array}$ & $\begin{array}{c}\text { Condition of existence of } \\
\text { the correlative link: } \\
\text { A }>50 \% \varrho>0.87\end{array}$ & $\mathbf{P}$ \\
\hline $\begin{array}{c}\text { Glycemia } \\
(\mathrm{N}=73)\end{array}$ & -0.097 & $-0.031-0.16$ & 0.5 & $\begin{array}{l}A<50 \% \\
|\varrho|<0.87\end{array}$ & $<0.05$ \\
\hline $\begin{array}{c}\text { Balanced } \\
\text { glycemia } \\
\text { glyc } \leq 1,10 \mathrm{~g} / \mathrm{L} \\
(\mathrm{N}=29)\end{array}$ & -0.057 & $-0.026-0.14$ & 0.2 & $\begin{array}{l}A<50 \% \\
|\varrho|<0.87\end{array}$ & $>0.05$ \\
\hline $\begin{array}{c}\text { Hyperglycemia } \\
1,10<\text { Glyc }<1,26 \mathrm{~g} / \mathrm{L} \\
(\mathrm{n}=8)\end{array}$ & +0.975 & $0.85-1$ & 77.7 & $\begin{array}{l}A>50 \% \\
\varrho>0.87\end{array}$ & $<0.05$ \\
\hline $\begin{array}{c}\text { Unbalanced } \\
\text { glycemia } \\
\text { Glyc } \geq 1,26 \mathrm{~g} / \mathrm{L} \\
(\mathrm{n}=36)\end{array}$ & +0.023 & $-0.026-0.066$ & 0.1 & $\begin{array}{l}A<50 \% \\
\varrho<0.87\end{array}$ & $>0.05$ \\
\hline
\end{tabular}

P: significance; HHcy: hyperhomocysteinemia; @: Spearman correlation coefficient; CI: confidence interval; A: correlation coefficient improvement expressing the presumption of the correlative link $\left(A=[1-\sqrt{ }((1-\varrho 2))]^{* 100)}[10]\right.$. 
Table 4. Correlation between the hyperhomocysteinemia and the lipids.

\begin{tabular}{|c|c|c|c|c|c|}
\hline HHcy & \multicolumn{2}{|c|}{ @ 95\% CI } & $\begin{array}{c}\text { A }(\%) \\
\text { (Presumption } \\
\text { of the link of } \\
\text { correlation) }\end{array}$ & $\begin{array}{c}\text { Condition of existence of the } \\
\text { correlative link: } \\
\text { A }>50 \% \\
\varrho>0.87\end{array}$ & $\mathrm{P}$ \\
\hline $\begin{array}{l}\text { Triglycerides } \\
\qquad(n=73)\end{array}$ & -0.132 & $-0.055-0.207$ & 0.9 & $\begin{array}{l}A<50 \% \\
|\varrho|<0.87\end{array}$ & $<0.05$ \\
\hline $\begin{array}{c}\text { Total Cholesterol } \\
\qquad(\mathrm{n}=73)\end{array}$ & -0.124 & $-0.045-0.195$ & 0.8 & $\begin{array}{l}A<50 \% \\
|\varrho|<0.87\end{array}$ & $<0.05$ \\
\hline $\begin{array}{l}\text { LDL Cholesterol } \\
\qquad(\mathrm{n}=73)\end{array}$ & -0.105 & $-0.031-0.169$ & 0.6 & $\begin{array}{l}A<50 \% \\
|\varrho|<0.87\end{array}$ & $<0.05$ \\
\hline $\begin{array}{l}\text { HDL Cholesterol } \\
\qquad(\mathrm{n}=73)\end{array}$ & +0.107 & $0.038-0.182$ & 0.1 & $\begin{aligned} A & <50 \% \\
\varrho & <0.87\end{aligned}$ & $<0.05$ \\
\hline
\end{tabular}

P: significance; HHcy: hyperhomocysteinemia LDL: low density lipoprotein cholesterol; HDL: high density lipoprotein cholesterol; @: Spearman correlation coefficient; A: correlation coefficient improvement expressing the presumption of the correlative link $(\mathrm{A}=$ $[1-\sqrt{ }((1-\varrho 2))]^{* 100)[10] . ~}$

\section{Discussion}

The hyperhomocysteinemia is an independent cardiovascular risk factor [11]. It is associated with the development and the accentuation of the cardiovascular complications of the type 2 diabetes [5]. In this regard, the exploration of the correlation between the hyperhomocysteinemia, the glycemia and the lipids was necessary. In order to appreciate the effect of independence or synergy of the hyperhomocysteinemia vis-a-vis the metabolism of carbohydrates and lipids, during the development of the cardiovascular diseases of type 2 diabetes. And also, in view of a better nutritional and clinical management of the type 2 diabetics in Côte d'Ivoire.

The relational study between the hyperhomocysteinemia and the duration of the type 2 diabetes has revealed a very strong correlation, with a correlative presumption of $99.80 \%$. That suggests that the hyperhomocysteinemia is strongly correlated with the duration of the type 2 diabetes. The duration of the diabetes seems to be a factor of increasement of the hyperhomocysteinemia. Our study is corroborated by those of Hultberg et al., [12] and Drzewoski et al., [13]. Besides, the hyperhomocysteinemia appears to be positively correlated with the glycated hemoglobin in subjects with unbalanced glycemia [13]. However, according to Hoogeveen et al., [14], when the glycemia is balanced, the correlation of the hyperhomocysteinemia to the glycated hemoglobin disappears.

So, we had explored the correlative link between the hyperhomocysteinemia and the glycemia in a general context; without distinguishing whether the glycemia was balanced or unbalanced. The correlation was negative and very low, even if it was significant $(\varrho=-0.097$; correlation link presumption: $0.5 \%$ ). We had concluded to an absence of a correlative link between the hyperhomocysteinemia and the glycemia in the general context. The diabetes and the hyperhomocysteinemia appear to be independent factors. Our result is in agreement with that of Laraqui et al., [15]. The average glycemic in our diabetics is $1.53 \pm 0.81 \mathrm{~g} / \mathrm{L}$; with a glycemia minimum of $0.78 \mathrm{~g} / \mathrm{L}$ and a glycemia maximum of $4.63 \mathrm{~g} / \mathrm{L}$. Our sample contained a mixture of diabetics, whose glycemia was either balanced or unbalanced.
Most of the diabetics had a balanced glycemia. These diabetics were regularly monitored on the nutritional level at the Nutrition Center of the National Institute of Public Health in Abidjan, and clinically at the Diabetic Center of Abidjan. The lack of correlation between the hyperhomocysteinemia and the glycemia could be explained by the effect of clinical and nutritional treatment in our diabetics. The medication had a corrective effect on the glycated hemoglobin, which is a precursor to the formation of the advanced glycation end products (AGE) [16]. The glycated hemoglobin and the AGEs produce free radicals [16]. The nutritional management brings a strengthe-ning of plasma B vitamins, but also the antioxidants against the propagation of the free radicals produced by the glycated hemoglobin, the AGEs and the hyperhomocysteinemia. Thus, the clinical and nutritional management contribute to balance the glycemia and regulate the homocysteinemia. Hence the absence of correlation between the hyperhomocysteinemia and the glycemia.

To check under the form of hypothesis, the assertion of Hoogeveen et al., [14] according to that "when is glycemia equilibrates, the correlation of the hyperhomocysteinemia to the glycemia disappears" we had selected only diabetics having a balanced (glycemia $\leq 1.10 \mathrm{~g} / \mathrm{L}$ ). This test has showed that the correlation between the hyperhomocystei-nemia and the glycemia was negative, low and non-significant $(\varrho=-0.097$; correlation link presumption: $0.5 \%$ ). We have concluded that there was no correlative link between the hyperhomocysteinemia and the balanced glycemia. Thus, our study has confirmed that of Hoogeveen et al., [14].

We had also checked under the form of hypothesis, the assertion of Drzewoski et al., [13] according to that "when the glycemia is unbalanced, the hyperhomocystei-nemia is correlated to the glycemia". So, we had selected only diabetics with a very unbalanced glycemia (glycemia $\geq 1.26 \mathrm{~g} / \mathrm{L}$ ). Our study has found a low positive, but not significant correlation between the hyperhomocysteinemia and the diabetes $(\varrho=+0.023$; correlation link presumption: $0.1 \%$ ). We had concluded to an absence of correlation between the hyperhomocysteinemia and the unbalanced glycemia. Our study did not confirm that of Drzewoski et al., [13], which suggested that "the supposed correlative link between the 
hyperhomocysteinemia and the glycemia" during the imbalanced glycemia spanned at the entire glycemia period $\geq 1.10 \mathrm{~g} / \mathrm{L}$. It was rather when the glycemia was regulated to the state of hyperglycemia $(1.10<$ glycemia $<1.26 \mathrm{~g} / \mathrm{L})$, that we had observed a significant correlation between the hyperhomocysteinemia and the hyperglycemia $(\varrho=+0.975$; correlation link presumption: $77.70 \%$ ). We had then concluded to the existence of a positive correlative link between the hyperhomocysteinemia and the hyperglycemia. That suggested to a strong synergistic interaction between the metabolisms of homocysteine and glucose during the period of the hyperglycemia. The hyperhomocysteinemia "would settle" in the diabetes probably during the period of the hyperglycemia and not in glycemia $\geq 1.26 \mathrm{~g} / \mathrm{L}$.

In terms of clinical management of diabetes, this would mean that it would be imperative for the clinician (nutritionist or doctor) to balance the diabetic glycemia. In fact, an unbalanced glycemia that would persist in hyperglycemia would be a favorable condition for the insidious installation of hyperhomocysteinemia in the type 2 diabetes. This recommendation is crucial because the hyperhomocysteinemia, (generally ignored in the treatment of diabetic) is associated with the development and accentuation of cardiovascular complications of type 2 diabetes. The quality of the glycemic balance and the duration of the diabetes can influence the effect of the hyperhomocysteinemia in the development of the type 2 diabetes cardiovascular diseases.

Several studies have established a link between the hyperhomocysteinemia and the lipids, because the hyperhomocysteinemia could promote the atherosclerosis involved in the cardiovascular diseases [17]. Several studies have also shown that the hyperhomocysteinemia promotes the increase of the atherogenic lipids (triglycerides, LDL, total cholesterol) and reduces the anti-atherogenic lipids (HDL) [7]. Thus, the study of Qujeq et al., [18] in 121 patients with myocardial infarction has showed a very strong negative correlation $(\varrho=$ -0.95) between the hyperhomocysteinemia and the HDLs and a very strong significant positive correlation $(\varrho=+0.98)$ between the hyperhomocysteinemia and the LDLs. The study of Anan et al., [19] in 40 Japanese diabetic subjects has showed that the hyperhomocysteinemia had a strong positive correlation with the triglycerides and a strong negative correlation with the HDLs. The study of Momin et al., [7] has also shown that the hyperhomocysteinemia had a positive correlation with the triglycerides and a negative correlation with the HDLs. Several studies have sustained that the hyperhomocysteinemia affected the lipid metabolism by several mechanisms such as: the down regulation of the HDLs and the conversion of the phosphatidylcholine to phosphatidylethanolamine which leads to the increased of the triglycerides [7].

In contrast, in our study, the correlations between the hyperhomocysteinemia and the atherogenic lipids (triglycerides, LDL and total cholesterol) are negative, significant, but very low. Also the positive correlation between the hyperhomocysteinemia and the anti-atherogenic lipids (HDL) is certainly significant, but very low. We have therefore concluded that there was no correlation between the hyperhomocysteinemia and the lipids. Our study is in agreement with those of De-Luis et al., [20] and Yadav et al., [21] who also had found no correlative link between the lipids and the hyperhomocysteinemia. Corroborating our study, Momin et al., [7] also have found that the total and
LDLs cholesterol tended to decrease in the presence of the hyperhomocysteinemia. The hyperhomocysteinemia can cause an oxidative stress (free radicals) that can entrave the production of the lipoproteins $[7,22]$.

The studies examining the correlation between the hyperhomocysteinemia and the lipids are mitigated [7]. Most of the studies have biases or confounding factors. Thus, the study of Momin et al., [7] had excluded from its cohort all subjects under anti-lipid treatment in order to observe a significant correlative link between the hyperhomocysteinemia, the triglycerides and the HDLs. In our study, the absence of a correlative link between the lipids and the hyperhomocysteinemia could partly be explained by the fact that our diabetics are for the most part under anti-lipid and dietetic treatment. That suggests that a correlative link could well be observed in our study if we had excluded all subjects under anti-lipid treatment from our diabetic cohort. Such an experiment may be the subject of a future study.

\section{Conclusion}

The hyperhomocysteinemia is strongly correlated with the duration of the type 2 diabetes. The study did not show a correlative correlation with the hyperhomocysteinemia and the glycemia, both in the context of balanced glycemia or unbalanced glycemia. However, when the glycemia is regulated to the state of hyperglycemia, a strong correlation was observed between the hyperhomocysteinemia and the glycemia. The study showed an absence of correlative link between the hyperhomocysteinemia and the lipids in our diabetics under anti-lipid treatment. The quality of the glycemic and lipid balance and the duration of the diabetes can influence the effect of the hyperhomocysteinemia in the development of the cardiovascular diseases of type 2 diabetes.

\section{References}

[1]. Peyrin-Biroulet L. Homocysteinemia, a new marker of severity in IBD ?. J Gastrointest Oncol. 2007 Mar 1;14(2):149-56.

[2]. Quillard M, Berthe MC, Sauger F, Lavoinne A. Plasma determination of homocysteine on CPC Immulite 2000: comparison with determination on IMX Abbott. Ann Biol Clin (Paris). 2003 Nov-Dec;61(6):699-704. PubMed PMID: 14711612.

[3]. Demets JP. A study by Harvard University researchers showed that too much homocysteine in the blood has a risk factor that can lead to cardiovascular disorders. But what is homocysteine? [Internet]. 2017 [cited 2017 Apr 17]. Available from: http://www.masantenaturelle.com

[4]. Grunitzky K, Balogou K, Bark-kombate R, Kombate D, Amouzou K. Homocysteinemia and ischemic strokes at the CHU Campus of Lomé. [Afr. J Neurol Sci. 2008;27(2).

[5]. Vangelder E, Delecourt F, Cardozo MB, Dhondt JL, Forzy G. Hyperhomocysteinemia and Type 2 Diabetes. Ann Clin Microbiol Antimicrob. 2006 Sep 1;64(5):485-489.

[6]. Guldener CV, Stehouwer CD. Homocysteine and vascular complications of diabetes. Diabetes Voice. 2003;48:31-33.

[7]. Momin M, Jia J, Fan F, Li J, et al. Relationship between plasma homocysteine level and lipid profiles in a community-based Chinese population. Lipids Health Dis. 2017 Mar 14;16(1):54. PubMed PMID: 28288621.

[8]. Yapo AE, Assayi M, et al. The reference values of 21 biochemical blood constituents of adult Ivorian presumed healthy. Afr Med Pub. 1990;(110):4957.

[9]. Faeh D, Chiolero A, Paccaud F. Homocysteine as a risk factor for cardiovascular disease: should we (still) worry about it?. Swiss Med Wkly. 2006 Dec 2;136(47-48):745-56. PubMed PMID: 17225194.

[10]. Labrousse C. Statistics: corrected exercises with course reminders. Dunod; 1978.

[11]. Demuth K. Homocysteine and cardiovascular prevention in the elderly. Ann Biol Clin (Paris). 2001 Jul 24;59(4):465-8.

[12]. Hultberg B, Agardh CD, Agardh E, Lövestam-adrian M. Poor metabolic 
control, early age at onset, and marginal folate deficiency are associated with increasing levels of plasma homocysteine in insulin-dependent diabetes mellitus. A five-year follow-up study. Scand J Clin Lab Invest. 1997 Jan 1;57(7):595-600.

[13]. Drzewoski J, Czupryniak L, Chwatko G, Bald E. Hyperhomocysteinemia in poorly controlled type 2 diabetes patients. Diabetes Nutr Metab. 2000 Dec;13(6):319-24. PubMed PMID: 11232756.

[14]. Hoogeveen EK, Kostense PJ, Beks PJ, Mackaay AJ, Jakobs C, Bouter LM, Heine RJ, Stehouwer CD. Hyperhomocysteinemia is associated with an increased risk of cardiovascular disease, especially in non-insulin-dependent diabetes mellitus: a population-based study. Arterioscler Thromb Vasc Biol. 1998 Jan;18(1):133-8. PubMed PMID: 9445267.

[15]. Laraqui A, Bennouar N, Meggouh F, et al. Homocysteine, lipoprotein (a): risk factors for coronary heart disease. Ann Biol Clin (Paris). 2002 SepOct;60(5):549-57. PubMed PMID: 12368140.

[16]. Hegab Z, Gibbons S, Neyses L, Mamas MA. Role of advanced glycation end products in cardiovascular disease. World J Cardiol. 2012 Apr 26;4(4):90102. PubMed PMID: 22558488.

[17]. Jarir J, Mohammadi H, Benazzouz B, Naamane A, et al. Study of Homocysteine and Lipid Metabolism in Moroccan Children with Type I Diabetes. Eur J Sci Res. 2012 Oct; 89 (4): 542-54.

[18]. Qujeq D, Omran TS, Hosini L. Correlation between total homocysteine, low-density lipoprotein cholesterol and high-density lipoprotein cholesterol in the serum of patients with myocardial infarction. Clin Biochem. 2001 Mar;34(2):97-101. PubMed PMID: 11311217.

[19]. Anan F, Masaki T, Umeno Y, Yonemochi H, Eshima N, et al. Correlations between homocysteine levels and atherosclerosis in Japanese type 2 diabetic patients. Metabolism. 2007 Oct;56(10):1390-5. PubMed PMID: 17884450

[20]. De Luis DA, Fernandez N, Arranz ML, Aller R, Izaola O, Romero E. Total homocysteine levels relation with chronic complications of diabetes, body composition, and other cardiovascular risk factors in a population of patients with diabetes mellitus type 2. J Diabetes Complications. 2005 Jan-
Feb;19(1):42-6. PubMed PMID: 15642489.

[21]. Yadav AS, Bhagwat VR, Rathod IM. Relationship of plasma homocysteine with lipid profile parameters in ischemic heart disease. Indian J Clin Biochem. 2006 Mar;21(1):106-10. PubMed PMID: 23105579.

[22]. Thampi P, Stewart BW, Joseph L, Melnyk SB, Hennings LJ, Nagarajan S. Dietary homocysteine promotes atherosclerosis in apoE-deficient mice by inducing scavenger receptors expression. Atherosclerosis. 2008 Apr;197(2):620-9. PubMed PMID: 17950295.

[23]. Zittoun J. Homocysteine and vascular pathology. Hematol. 1998 Jan 27; 4(1):7-16.

[24]. Rais L, Wafi M, Lahbil D, Iraki M, et al., Ocular and systemic complications of homocystinuria: a report of five cases. J Fr Ophtalmol. 2003 Dec;26(10):1045-50. PubMed PMID: 14691398.

[25]. Houston M. Homocysteine-a risk factor for vascular diseases: Guidelines for the clinical practice. J Am Nutra Assoc. 2004;7(1):11-21.

[26]. Girs N, Giet D. Is the dosage of homocysteine relevant to the General Practitioner ?. Med J Lig. 2006;61(5-6):352-61.

[27]. Booth GL, Wang EE. Preventive health care, 2000 update: screening and management of hyperhomocysteinemia for the prevention of coronary artery disease events. CMAJ. 2000 Jul 11;163(1):21-9. PubMed PMID: 10920726.

[28]. Stanger O, Hermann W, Pietrzik K, Fowler B, Geisel J, Weger M. 2012. Consensus Article of DACH-Liga Homocysteine on the Rational Clinical Use of Homocysteine, Folic Acid and B vitamins in cardiovascular and thrombotic diseases. Guidelines and Recommendations [Internet]. 2017 [cited 2017 Mar 20]. Available from: http://www.dach-liga-homocystein.org.

[29]. Mishra PK, Tyagi N, Sen U, Joshua IG, Tyagi SC. Synergism in hyperhomocysteinemia and diabetes: role of PPAR gamma and tempol. Cardiovasc Diabetol. 2010 Sep 9;9:49. PubMed PMID: 20828387.

[30]. Friedewald WT, Levy RI, Fredrickson DS. Estimation of the concentration of low-density lipoprotein cholesterol in plasma, without use of the preparative ultracentrifuge. Clin Chem. 1972 Jun;18(6):499-502. PubMed PMID: 4337382 . 